\title{
Error characterization of sea surface salinity products using triple collocation analysis
}

\author{
N. Hoareau, M. Portabella, W. Lin, J. Ballabrera-Poy, and A. Turiel.
}

\begin{abstract}
The Triple Collocation (TC) technique allows the simultaneous calibration of three independent, collocated data sources, while providing an estimate of their accuracy. In this study, the TC is adapted to validate different salinity data products along the tropical band. The representativeness error (the true variance resolved by the relatively high-resolution systems but not by the relatively low-resolution system) is accounted for in the validation process. A method based on the intercalibration capabilities of TC is used to estimate the representativeness error for each triplet, which is found to impact between $15 \%$ and $50 \%$ the error estimation of the different products. The method also sorts the different products in terms of their resolving spatio-temporal scales. The six salinity products (sorted from smaller to larger scales) used here are: the in-situ data from the Global Tropical Moored Buoy Array (TAO), the GLORYS2V3 ocean reanalysis output provided by Copernicus, the satellite-derived Aquarius Level 3 version 4 (AV4) and SMOS Objectively Analyzed (SOA) maps, and the climatology maps provided by the World Ocean Atlas (WOA). This validation approach aims to assess the quality of the different salinity products at the satellite-resolved spatiotemporal scales. The results in the tropics for 2013 show that, at the AV4 resolved scales, the Aquarius product has an error of 0.17 , and outperforms TAO, GLORYS2V3 and the SMOS Objectively Analysis maps (SOA). However, at the SOA resolved scales (which are coarser than those of the Aquarius product because of the large OA correlation radii used), the SMOS product has an error of 0.20 , slightly lower than that of GLORYS2V3, Aquarius, and TAO. The WOA products show the highest errors. Higher order calibration may lead to a more accurate assessment of the quality of the climatological products.
\end{abstract}

Index Terms-Remote sensing, sea measurements, error analysis.

This paragraph of the first footnote will contain the date on which you submitted your paper for review. This work was supported by the Spanish $\mathrm{R}+\mathrm{D}$ plan under projects PROMISES (ESP2015-67549-C3-2) and L-BAND (ESP2017-89463-C3-1-R).

N. Hoareau is with Institut de Ciènces del Mar, CSIC, Departament d'Oceanografia Física i Tecnològica, Barcelona, Spain (e-mail: nhoareau@icm.csic.es).

M. Portabella is with Institut de Ciènces del Mar, CSIC Departament d'Oceanografia Física i Tecnològica, Barcelona, Spain (e-mail: portabella@icm.csic.es).

W. Lin is with School of Marine Sciences, Nanjing University of Information Science and Technology (NUIST), Ningliu Road 219, Pukou, Nanjing, 21004, China (e-mail: wenminglin@nuist.edu.cn).

J. Ballabrera is with Institut de Ciènces del Mar, CSIC, Departament d'Oceanografia Física i Tecnològica, Barcelona, Spain (e-mail: joaquim@icm.csic.es).

A. Turiel is with Institut de Ciènces del Mar, CSIC Departament d'Oceanografia Física i Tecnològica, Barcelona, Spain (e-mail: Turiel@icm.csic.es).

\section{INTRODUCTION}

$\mathrm{T}$ HE monitoring of the global distribution of sea-surface salinity (SSS) is vital to understand the oceans' role in Earth's climate. Until the advent of the spaceborne L-band radiometers, SSS observations were mainly acquired by in situ sensors (moored buoys, drifters, and thermosalinographs). As a result, knowledge of the spatial and temporal variability of salinity had been scarce due to the lack of a comprehensive set of salinity observations. While in situ data (e.g., Argo floats) were being used in a growing number of studies (for example, [1], [2], or [3]), numerical models were also widely used as a complementary source of such information, e.g. [4] and references therein.

The spatio-temporal resolution achieved by satellite salinity measurements has no equivalent among the other existing salinity observation systems. Since the launch of the Soil Moisture and Ocean Salinity (SMOS) mission (2009) and then the Aquarius mission (2011), more than seven years of satellite-derived SSS data, with a spatial and temporal resolution adequate for climate and ocean general circulation studies, have become available. The L-band radiometers onboard SMOS and Aquarius have proven to be challenging notably in the case of the SMOS interferometric measurements. While there have been notable efforts to improve the quality of retrievals [5], [6], L-band radiometry is still in its infancy, and the quality of derived salinity products is expected to improve with time. Currently, higher-level processing efforts, i.e., various spatial and temporal averaging and data fusion techniques, have been implemented to better recover structured and meaningful geophysical information from remote sensing SSS retrievals [7], [8], [9].

In all cases a comprehensive validation is essential to characterize the information provided by these products. For example, [10] present a novel technique, the so-called singularity power spectra, or SPS, to analyze the geophysical consistency of the different satellite-derived SSS maps. In particular, the SPS was able to reveal the small-scale turbulent cascade signature, and thus demonstrated that most of the studied SSS products were able to represent a reasonable spatial variability. Besides geophysical consistency, it is of utmost importance to estimate the uncertainty associated to each SSS dataset.

Direct comparisons against in situ salinity measurements have been extensively used to assess the reliability of remotely sensed SSS [11], [12], [13], [14]. Indeed, such direct 
comparisons provide relevant information about the quality of the different datasets. For example, [15] identified corrupted buoy time series (due to, e.g., instrument malfunctions), by directly comparing them to other in situ and satellite-based SSS products. Such validation approach, however, has some limitations as in situ data are assumed to be true or perfect. Indeed, although the in situ instrument errors are usually considered negligible, in situ measurements include spatial and temporal scales of variability that differ from remotesensed measurements or ocean model outputs. For example, an in situ observation, e.g., Argo, represents an almost instantaneous and point measurement, whereas data products, e.g., satellite or model output, are given in grids with spatial sizes of 10-100 km, with temporal averages of 1-30 days. Moreover, satellite SSS products measure the top $1 \mathrm{~cm}$ of the ocean surface while in situ SSS measurements are usually obtained few meters below the surface. All of these differences result in the presence of a representativeness error [16], [17], [18], [19], which must be taken into account during validation.

The so-called triple collocation (TC) technique, first introduced by [16] to estimate the uncertainties of three sea surface wind data sources, is being adapted and used in this paper to estimate the uncertainties of different SSS data sources (i.e., in situ, satellites, and numerical ocean model output). In this work, we will use a set of collocated measurements from three different SSS data sources, one source is assumed to be calibrated (called as the reference). In order to estimate the random measurement error of all three sources and the relative linear calibration coefficients of two sources with respect to the reference one. To do so, we need to properly estimate the representativeness error, which was defined by [16] as the short-scale true variance resolved by the relatively high-resolution systems but unresolved by the coarser-resolution system.

The TC method has been used for assessing the quality of a wide variety of geophysical variables, including sea surface winds [16], [17], [18], [19], soil moisture [20], [21], and precipitation [22], among others. A simplified version of TC, in which the representativeness error was not considered, has been used to assess the quality of several SSS products [23]. In this paper, the importance of considering the representativeness error is highlighted. The method developed by [24] to estimate sea surface wind errors in high wind variability conditions is adopted to properly estimate such error and thus provide accurate measurement errors for different SSS data products (including, in situ, ocean reanalysis, satellite, and climatology) at the spatio-temporal scales resolved by the satellite data.

In section II, a description of the different SSS data products to be validated is provided. In section III, the TC technique as well as its adaptation for SSS validation purposes and the method to estimate the representativeness error, are presented. The TC results are thoroughly analyzed in section IV. Finally, the concluding remarks and the outlook are discussed in section V.

\section{SSS PRODUCTS}

\section{A. Data}

The period of this study corresponds to the year 2013. This period has been chosen for two reasons: i) All the SSS data sources used are available in this period; ii) the year 2013 is not influenced by strong events like El Niño (2014-2015) or La Niña (2011-2012), which are known to be unresolved by the climatology, thus leading to strong biases in the latter.

In situ data: The in situ sea surface salinity time series come from the National Oceanic Atmospheric Administration (NOAA) Tropical Ocean Atmosphere (TAO) buoy arrays in the tropical Pacific, the Japan Agency for Marine-Earth Science and Technology (JAMSTEC) Triangle Trans-Ocean Buoy Network (TRITON) buoys in the western Pacific, the Prediction and Research Moored Array in the Atlantic (PIRATA), and the Research Moored Array for AfricanAsian-Australian Monsoon Analysis and Prediction (RAMA) in the tropical Indian Ocean. Only data measured at 1-1.5m depth with the highest quality flag value (i.e., 1 and 2) have been used in this study. The data can be found at https://www.pmel.noaa.gov/ and are served at daily scale. The location of the 28 different buoy arrays retained is shown in Figure 1. Note that salinity is defined using the Practical Salinity Scale 1978 [25], and salinity values are given without unit.

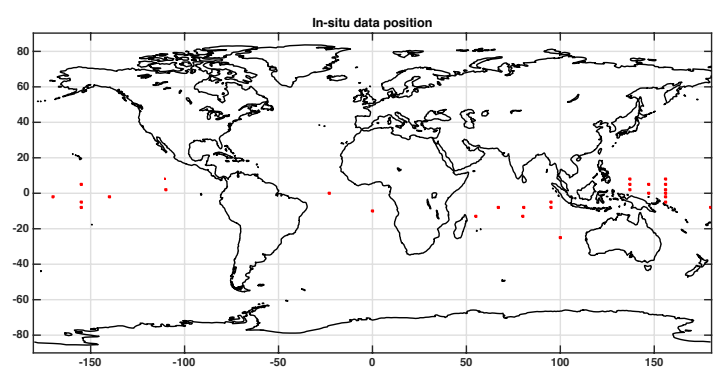

Fig. 1. Location (red symbols) of the TAO, PIRATA and RAMA buoy arrays used in this study.

SMOS: The SMOS satellite, launched in November 2009 as part of the European Space Agency's (ESA) Living Planet Program [26], is the first polar-orbiting satellite with a passive microwave sensor operating in the L-Band $(1.4 \mathrm{GHz})$. Using polarimetric interferometry from 69 individual radiometers, SMOS retrieves SSS in a $1200 \mathrm{~km}$-wide field of view, providing global coverage every three days. ESA's Level-2 (L2) swath-based SSS products have a spatial resolution of 30$50 \mathrm{~km}$, but a rather low accuracy of 0.6 to 1.7 [11], [12].

The Barcelona Expert Centre (BEC) serves different SMOS SSS high-level products, with different spatial and temporal resolutions. In this study, the operational v2.0 SMOS objective analysis (SOA) product is used [27]. This product has been 
generated from the v6.22 version of the SMOS SSS Level 2 products provided by ESA. Additionally, the Land Sea Contamination has been mitigated by means of the empirical salinity debiasing method proposed by [9]. In the OA scheme, no auxiliary SSS data have been used as background, using a constant background value of 35 instead. Also note that in order to mitigate the relatively poor radiometric accuracy of the SMOS measurements, the scheme uses large correlation radii, i.e., $321 \mathrm{~km}, 267 \mathrm{~km}$, and $175 \mathrm{~km}$ (as in [28]). The SOA product, with a 0.25 spatial grid and 9-day temporal resolution, is served at daily frequency. Further details on this and other products distributed by BEC can be found at http://bec.icm.csic.es/.

Aquarius: The Aquarius instrument on-board the Aquarius/SAC-D satellite was the result of a collaborative effort between the National Aeronautics and Space Agency (NASA) and the Argentine Space Agency Comisión Nacional de Actividades Espaciales (CONAE). It operated from its launch in June 2011 until June 2015. The instrument consisted of three real-aperture L-band $(1.4 \mathrm{GHz})$ radiometers in a push broom configuration at 29-, 38-, and 46-degrees incidence angles, with footprints sizes of $76 \mathrm{~km}$ (along-track) x $94 \mathrm{~km}$ (cross-track), $84 \mathrm{~km} \mathrm{x} 120 \mathrm{~km}$ and $96 \mathrm{~km} \times 156 \mathrm{~km}$, respectively, yielding a combined swath width of $370 \mathrm{~km}$. The platform also carried a scatterometer, operating at $1.26 \mathrm{GHz}$, which measured the ocean backscatter in each footprint. The scatterometer was used for correcting sea surface roughness effects in the TB measurements, thus improving salinity retrievals. The version 4 of the Aquarius Level 3 SSS product (AV4) is distributed with one-degree grid resolution and 7-day temporal resolution, and was created from version 4 of the Aquarius/SCA-D operational processor. The data are available at ftp://podaac-ftp.jpl.nasa.gov/allData/aquarius/L3/mapped/

GLORYS2V3 Reanalysis: The Mercator Ocean Global Ocean Physics Reanalysis GLORYS2V3 1993-2013, available from the Copernicus Marine Environmental Monitoring Service (CMEMS), http://marine.copernicus.eu, uses the NEMO [29] version 3.1 ocean model in the ORCA025_LIM configuration. Its spatial and temporal resolution is 0.25 degree and daily average, respectively. See [30].

World Ocean Atlas climatology: The three-dimensional monthly salinity maps from the National Oceanic and Atmospheric Administration's (NOAA) World Ocean Atlas 2013 (WOA13) [28] and 2009 (WOA09) [31], created by optimal interpolation of all the available historical sets of in situ data, are used. The horizontal grid resolution is 0.25 degree for WOA13 and a daily interpolation has been applied to create a daily climatology.

\section{B. Collocation Procedure}

The above-mentioned satellite, model, and climatological SSS products have been collocated with the in situ data. The closest grid point to the in situ location is used. All products but AV4 are served daily or interpolated at daily scales. As such, only collocations every 7 days (i.e., AV4 temporal resolution) are considered. Note that the monthly climatology (WOA13) has been interpolated at daily scales for collocation purposes, although its actual temporal resolution does not change. As such, all the (daily) salinity products are temporally collocated to the central day (i.e., day 4) of each AV4 map. As a consequence, all the different triple collocation combinations used in section IV correspond to the same time periods and locations, i.e., the TAO, GLORYS2V3, AV4, SOA, WOA09, and WOA13 datasets are all collocated together (rather than triplets, sextuplets are obtained). A total of 1456 collocations are obtained over the study period, i.e., 2013.

Table 1 shows the spatial and temporal characteristics of the different SSS products used in this study. In summary, the following temporal and spatial resolutions are used in descending order: 1) buoy data at daily and pointmeasurement level; 2) GLORYS2V3 at daily and 0.25 degree resolution; 3) satellite (AV4 and SOA) at roughly weekly and 0.25 to 1 degree resolution; and 4) climatology (WOA09 and WOA13) at monthly and 0.25 to 1 degree resolution.

The work of [32] shows that the temporal averaging is equivalent to the spatial averaging in terms of the resulting signal variance (Taylor approximation). That is, the temporal averaging induces a reduction of the true signal variance, which is equivalent to that induced by the spatial averaging. In this study (see Table 1) we use collocated data products of different spatial and temporal resolutions. The latter are interpreted in terms of the true variance resolved by each product, i.e., in terms of the (joint) spatio-temporal scales (resolution) resolved by each product. For simplicity, the term spatio-temporal scale (resolution) is hereafter referred to as scale (resolution).

\begin{tabular}{c|c|c} 
& $\begin{array}{c}\text { Spatial resolution } \\
\text { (Grid size) }\end{array}$ & $\begin{array}{c}\text { Temporal } \\
\text { resolution }\end{array}$ \\
\hline $\boldsymbol{T A O}$ & Point & Daily average \\
\hline $\boldsymbol{G L O R Y S 2 V 3}$ & $0.25^{\circ}$ & Daily product \\
\hline $\boldsymbol{A} \boldsymbol{V 4}$ & $1^{\circ}$ & 7 days average \\
\hline WOA & $0.25^{\circ}$ & 9 days average \\
\hline WOA09 & $0.25^{\circ}$ & $\begin{array}{c}\text { Daily interpolation } \\
\text { from monthly product }\end{array}$ \\
\hline & $1^{\circ}$ & Monthly product
\end{tabular}

Table 1: Sea Surface Salinity products

The triple collocation method described in section III will help to: i) estimate what the representativeness errors of the different products are in order to compute the errors for each data product at the satellite scales; and ii) sort the products according to their effective resolution.

\section{TRIPle Collocation Methodology}

The triple collocation (TC) method was conceived by [16] as a tool for intercalibration and individual error assessment of three different collocated sea-surface wind datasets. In fact, 
TC is rather generic and can be applied to any geophysical variable and data product, provided that errors are additive, error distributions are close to Gaussian, and that the collocated data sources are independent. In this section, the method is briefly presented and adapted to SSS datasets.

TC uses three spatially and temporally collocated data sources of the variable of interest to solve a system of equations in an iterative way, leading to intercalibrated data sources and estimates of the error variance of each source. Given three measurement systems, $s_{i}, i=1,2,3$, that for example represent buoy, satellite, and climatology, respectively, the measurements and measurement errors are approximated by the following linear expression:

$$
s_{i}=a_{i} S+b_{i}+\delta_{i}
$$

where $\mathrm{S}$ is the common quantity in this study, i.e., the true salinity at the scales commonly resolved by all three data sources, $a_{i}$ and $b_{i}$ stand for the scaling and bias calibration coefficients, respectively, and $\delta_{i}$ for the random measurement error. $\delta_{i}$ is assumed to be unbiased, and its variance does not change with $S$. These assumptions hold well for the salinity variable if the pseudo-anomaly of $S$ is taken (see section III.A).

Equation (1) is solved iteratively under several assumptions. First, one data source (e.g., $i=1$ ) is used as the calibration reference, i.e. $a_{1}=1$ and $b_{1}=0$. Second, all random measurement errors are assumed to be uncorrelated with $S$. Now, let's suppose that systems 1 and 2 (e.g., buoy and remote sensing salinity) resolve smaller turbulent scales than system 3 (e.g., WOA13), and that the variance common to these smaller scales, $r^{2}=\left\langle\delta_{1} \delta_{2}\right\rangle$, is part of the measurement errors $\delta_{1}$ and $\delta_{2}$. By definition, $r^{2}$ is the correlated part of the representativeness errors of $s_{1}$ and $s_{2}$. Assuming that, since $s_{3}$ does not include these smaller scales, its measurement error $\delta_{3}$ is independent of $\delta_{1}$ and $\delta_{2}$, and $\left\langle\delta_{1} \delta_{3}\right\rangle=\left\langle\delta_{2} \delta_{3}\right\rangle=0$. In summary, salinity errors of different systems are all assumed to be uncorrelated, except for the spatial representativeness error [16], due to the turbulent scales only resolved by systems 1 and 2.

Note that system 1 (e.g., buoy) often resolves smaller scales than system 2 (e.g., satellite). If one would want to estimate the measurement errors at the scales resolved by system 1, another representativeness error $r^{\prime 2}$, i.e., the true variance resolved by system 1 but not by system 2 , needs to be estimated. However, in this paper, the goal is to estimate the measurement errors of the different SSS data products at the satellite-resolved scales (i.e., system 2 or system 3, as shown in section IV), for which only $r^{2}$ needs to be estimated. Note also that when the estimated $r^{2}$ is found to be zero, it implies that system 2 resolves the same scales as system 3 .

The calibrated data sets are created by

$$
s_{i}^{\prime}=\frac{1}{a_{i}}\left(s_{i}-b_{i}\right), \quad i=2,3
$$

Note that $s_{1}^{\prime}=s_{1}$ by definition. The TC analysis is implemented using an iterative approach. The calibrated data sets $s_{i}^{\prime}, i \neq r e f$ are used to estimate the new calibration factors, until these parameters converge. Normally, the convergence is reached within six iterations. After calibration, the error variance of each salinity product, estimated at the scale of system 3 (e.g., WOA13), is given by

$$
\left\langle\delta_{i}^{2}\right\rangle=M_{i i}-\varepsilon^{2}
$$

The quantity $\varepsilon^{2}$ denotes the common true variance in the three measurement systems and has several different expressions. That is, after calibration the following relations hold: $\varepsilon^{2}=M_{12}-r^{2}=M_{23}=M_{13}$. In these equations $M_{i i}$ and $M_{i j}$ correspond to the second-order moment of system $i$ and the mixed second-order moment of systems $i$ and $j$, respectively. To obtain the error variances on the scale of system 2 (i.e., typically the satellite scale), $r^{2}$ has to be subtracted from both the system 1 (e.g., buoy) and the system 2 (e.g., satellite) error variances, and added to that of system 3 (e.g., WOA13).

In summary, in order to properly estimate the random errors of the different sources, one has to first define the "truth" in terms of its spatial (both vertical and horizontal) and temporal representation. The representativeness error is actually the true variance that an observation system resolves (does not resolve) but is not resolved (is resolved) by the defined "truth". In this study, the "truth" spatio-temporal representation is defined as the ocean salinity at $1 \mathrm{~cm}$ depth and at the satellite-resolved (AV4 and SOA) spatio-temporal scales.

\section{A. Adaptation of TC to SSS: use of SSS pseudo-anomalies}

To perform TC with salinity data (which are centred around 35 for open ocean), one needs to centre the distribution around 0 to avoid numerical problems in the TC implementation. In what follows, we use system 2 to justify such need (although the derivation can be done for any of the three systems).

We define the measurement of SSS as

$$
s_{i}=\Delta s_{i}+K
$$

with $K=\left\langle s_{1}\right\rangle$ is the mean value of the reference measurement (system 1) and $\Delta s_{i}$ the pseudo-anomaly of the measurement system $i$, i.e., the deviation of the $s_{i}$ values from the constant $K$.

The mixed-second order moment between system 2 (e.g., satellite) and system 3 (e.g., climatology) becomes

$$
M_{23}=\left\langle s_{2} s_{3}\right\rangle=\left\langle\left(\Delta s_{2}+K\right)\left(\Delta s_{3}+K\right)\right\rangle
$$

and the second order moment for system 2 is

$$
M_{22}=\left\langle s_{2} s_{2}\right\rangle=\left\langle\left(\Delta s_{2}+K\right)\left(\Delta s_{2}+K\right)\right\rangle
$$

By using (4), (5), and (6), the error variance for system 2 is calculated as

$$
\left\langle\delta_{2}^{2}\right\rangle=M_{22}-M_{23}
$$




$$
\left\langle\delta_{2}^{2}\right\rangle=\left\langle\Delta s_{2}^{2}\right\rangle-\left\langle\Delta s_{3} \Delta s_{2}\right\rangle+K\left(\left\langle\Delta s_{2}\right\rangle-\left\langle\Delta s_{3}\right\rangle\right)
$$

After calibration, the third term of Eq. 7 is zero and therefore does not influence the final error variance value. However, in the first TC iteration, uncalibrated data are used and, as such, the third term (with $K \approx 35$ and $\Delta s_{3}>\Delta s_{2}$ ) can lead to negative $\left\langle\delta_{2}^{2}\right\rangle$ (error variances), which is impossible by definition. However, if TC is performed with $\Delta s_{i}$ instead of $s_{i}$, the salinity distributions of the three measurement systems are roughly centred on 0 , and in turn TC (i.e., equivalent to Eq. 7 but with $K=0$ ) is robust through all iterations.

In fact, the sea-surface wind TC analysis is performed for the zonal and meridional wind components [16], [18], [20], which have distributions around zero as well.

\section{B. Representativeness error estimation}

As already discussed in the previous sections, it is very important to accurately estimate the representativeness error $r^{2}$, i.e., the common true variance of systems 1 and 2 not resolved by system 3 , in order to successfully estimate the individual random errors of each of the three collocated data sources. Several techniques have been proposed over the last years to estimate $r^{2}$. In [17], $r^{2}$ is estimated by integrating the difference between the scatterometer wind power density spectra (PDS) and the numerical weather prediction (NWP) model output PDS from the finest scatterometer scale of 25 $\mathrm{km}$ to the largest ECMWF error scale of $800 \mathrm{~km}$. In [33], the cumulative variance was calculated as a function of the spatial scale, and the representativeness error was found to be given by the difference in cumulative variance of scatterometer and NWP wind components at a scale of $200 \mathrm{~km}$. In both approaches, it is necessary to process representative series of wind data of sufficient length in order to compute accurate wind spectra or cumulative variances. Moreover, as shown by [10], the PDS spectral slopes of the different SSS products are quite sensitive to the presence of noise, which is particularly high for SSS products. In this paper, the novel approach introduced by [19] is adopted to compute the representativeness error of SSS products.

As discussed at the beginning of section III, TC equations are solved iteratively by updating the calibration coefficients after each iteration and by applying them to the different data sources in the next iteration, see (2). A strong assumption of the TC method is that, once convergence is achieved, the three data sources are well intercalibrated. This can only be achieved with consistent calibration coefficients, $r^{2}$, and measurement error values, since all of them are directly related by the TC formulation. In particular, $r^{2}$ directly influences the scaling factor of system 3 (see [34] for further details). As such, setting a wrong $r^{2}$ value leads to a miscalibrated system 3 dataset, with respect to systems 1 and 2. Therefore, an effective way of estimating $r^{2}$ is to repeat the TC analysis for different $r^{2}$ values until an optimal intercalibration of the different data sources is achieved.

Figure 2 shows an example for the triple collocated datasets TAO-GLORYS2V3-SOA or TGS (hereafter, the following notation will be used for triple collocated datasets of system 1system 2-system 3) before and after TC. The top panels show the two-dimensional scatterplots of TAO and SOA, while the bottom panels show the scatterplots of GLORYS2V3 and SOA, before TC (left panels) and after TC (with a representativeness error of 0.023 , right panels). Note that the red dashed line represents the linear regression of the scatterplots. It is clear that TC leads to well intercalibrated SSS datasets, as denoted by the red-dashed lines on the right panels being closer to the diagonal (i.e., a slope close to 1) than those on the left panels. In this example, the best intercalibration between the three datasets (TAO, GLORYS2V3, and SOA) is achieved for $r^{2}=0.023$. As shown in section IV.A, for other $r^{2}$ values, TC leads to a (slightly) poorer intercalibration.
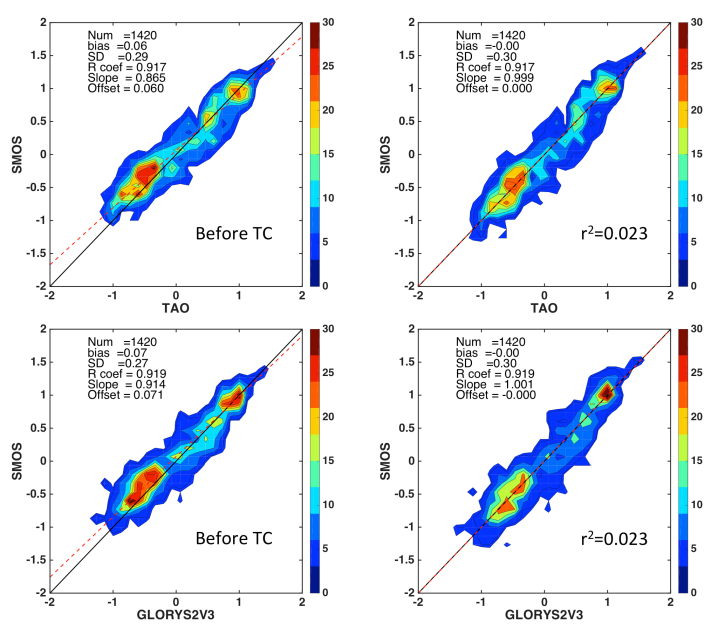

Fig. 2. Scatterplots of the SSS pseudo-anomalies from TAO (system 1, top) and GLORYS2V3 (system 2, bottom) versus SOA (system 3), before applying TC (left) and after applying TC with a representativeness error, $\mathrm{r}^{2}$, of 0.023 (right). The legend shows the number of data points (Num), the mean bias and the standard deviation (SD) of the scatterplot, and the regression coefficient ( $\mathrm{R}$ coef) together with the slope and offset.

\section{TRIPle COllocation Results}

As discussed in the previous sections, the goal of this analysis is to assess the quality of each individual SSS data product at the scales resolved by the satellite systems, i.e., Aquarius (AV4) and SMOS (SOA). It will be shown in section IV.A, the scales resolved by AV4 and SOA products differ. Therefore, the error assessment is presented in section IV.B at each scale separately.

By definition, the error assessment of each SSS product should lead to the same results regardless of the triplet combination used in TC, provided that the assessment is indeed carried out at the same scales. As such, in order to analyse potential inconsistencies and uncertainties of the TC method, several triplet combinations are analysed in this section: TAO-GLORYS2V3-AV4 (TGA), TAO-AV4-SOA (TAS), TAO-AV4-WOA09 (TA09), TAO-AV4-WOA13 
(TA13), TAO-GLORYS2V3-SOA (TGS), TAO-SOAWOA09 (TS09), and TAO-SOA-WOA13 (TS13). Note that in the TC iterative process, a 4-sigma test is performed. As a result of this quality control, about $2.5 \%$ of collocations are filtered out.

\section{A. Representativeness error}

Figure 3 shows the regression coefficient (slope) values after TC for different $r^{2}$ values and for two different triplets, i.e., TGA (left) and TAS (right). The solid (dashed) line indicates the slope of system 1 (system 2) versus system 3. Note that the estimated $r^{2}$ value corresponds to that obtained at the intersection of the horizontal dotted line (slope value of 1) and the solid/dotted lines, i.e., the $r^{2}$ value that leads to well intercalibrated datasets after TC (see section III.B). Both the solid and the dashed lines should intersect the dotted line at the same $r^{2}$ value. As seen in Figure 3, they intersect the dotted line at only slightly different $r^{2}$ values, indicating that the $r^{2}$ estimation method is indeed quite consistent. Since the value of $r^{2}$ for a particular triplet has to be unique, the average of both values (those obtained from the solid and dashed lines) is calculated and used as the estimated $r^{2}$.
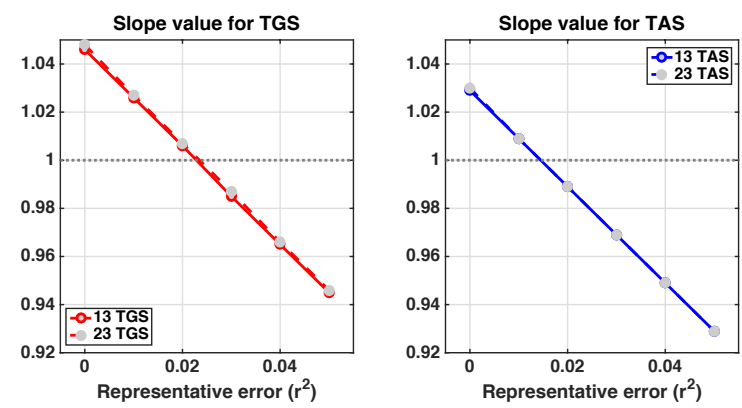

Fig. 3. Slope values as a function of the representativeness error $\left(\mathrm{r}^{2}\right)$ for the triplets TAO-GLORYS2V3-SOA (left) and TAO-AV4-SOA (right). The solid lines correspond to the slope values of the scatterplot system $1 /$ system 3 (TAO/SOA), while the dashed lines correspond to the slope values of the scatterplot system2/system3 (GLORYS2V3/SOA in red, and AV4/SOA in blue).

This approach for estimating $r^{2}$ also identifies the systems having the finest (systems 1 and 2) and the coarsest effective resolution (system 3). For example, when running the triple collocation algorithm for TAG or TSA (instead of TGA or TAS), the $r^{2}$ estimation does not converge, i.e., the solid and dashed lines never intersect the dotted line for positive $r^{2}$ values (not shown). This indicates that GLORYS2V3 is indeed of higher resolution than AV4, and that AV4 is in turn of higher resolution than SOA.

Therefore, the sorting of systems 1,2 , and 3 used in this analysis is not random, but already indicates the spatiotemporal scales of each product in ascending order. Note that TAO point measurements obviously represent the smallest scales and they are always used as system 1 . However, by construction, the TAO errors are estimated at the resolution of system 2 (see section III.B). Moreover, the larger the $r^{2}$ value, the larger are the scales resolved by system 3 (relative to those resolved by systems 1 and 2).

\begin{tabular}{c|c|c|c|c} 
& $\boldsymbol{T G A}(\boldsymbol{T G S})$ & $\boldsymbol{T A 1 3}(\boldsymbol{T S 1 3})$ & $\boldsymbol{T A 0 9}(\boldsymbol{T S} 09)$ & $\boldsymbol{T A S}$ \\
\hline $\boldsymbol{r}^{2}$ & $0.009(0.023)$ & $0.027(0.011)$ & $0.034(0.020)$ & 0.015
\end{tabular}

Table 2: Representativeness error $\left(\mathrm{r}^{2}\right)$ for the different triplets of SSS data: TAO-GLORYS-AV4 (TGA), TAO-GLORYS-SOA (TGS), TAO-AV4WOA13 (TA13), TAO-SOA-WOA13 (TS13), TAO-AV4-WOA09 (TA09), TAO-SOA-WOA09 (TS09) and TAO-AV4-SOA (TAS).

Table 2 shows $r^{2}$ for the different Aquarius-based triplets and, for comparison, the analogous SMOS-based triplets in parenthesis. According to the sorting of systems 2, and 3, and the magnitude of the estimated $r^{2}$ values, one can easily deduct that the sorting of the different products according to their spatio-temporal scales in ascending order is: GLORYS2V3 (see TGA and TGS), AV4 (see TAS, TA13, and TA09), SOA (see TS13 and TS09), WOA13 (see lower $r^{2}$ values for TA13 and TG13 than for TA09 and TG09), and WOA09.

The TGA, TGS, and TAS triplets show consistent results, i.e., the TGS $r^{2}$ value is almost equal to the sum of the TGA and TAS $r^{2}$ values. That is, the difference between GLORYS2V3 and SOA scales (represented by a true variance of 0.023 ) is almost identical to the sum of the difference between GLORYS2V3 and AV4 scales (0.009) and the difference between AV4 and SOA scales (0.015). This indicates that these four datasets comply with all TC assumptions discussed in section III (i.e., independent datasets, Gaussian distributions, effective linear calibration). It is important to note that the relatively large $r^{2}$ value of TAS $(0.015)$ indicates a noticeable difference between the AV4resolved scales and the SOA resolved scales. Although the Aquarius footprint is larger than that of SMOS, the SMOS product (SOA) clearly results in coarser spatio-temporal scales than AV4 since it uses large correlation radii in order to reduce the relatively poor radiometric accuracy of the SMOS measurements (see section II.A).

When looking at the triplets that include climatology, the results are also consistent. For example, the $r^{2}$ value for TA13 $(0.027)$ is similar to the sum of the TAS $(0.015)$ and the TS13 $(0.011)$ values. Moreover, the TS09 $r^{2}$ value $(0.034)$ is larger than that of TA09 (0.020), as expected since AV4 resolves smaller scales than SOA.

Figure 4 (left) shows the same as Figure 3 but for the triplet TA09. In contrast with Figure 3, the solid and the dashed lines in Figure 4 (left) are separated, leading to different intersections with the horizontal dotted line. This indicates that one cannot obtain a fully consistent $r^{2}$ value for this particular triplet. This is mainly due to the fact that there is no effective linear calibration for this particular triplet. This is shown by the scatterplot of WOA09 and AV4 (Figure 4, right), which corresponds to the output of TC using an $r^{2}$ of 0.034 (given by the average intersection of the solid and dashed lines with the dotted line in Figure 4, left). Although the distribution lies somewhat around the diagonal, it has an irregular shape, indicating the need for higher-order calibration. Since the TC 
assumes linear calibration, the uncertainty of the $r^{2}$ values (and corresponding random errors) from triplets that include climatology will be larger. However, as seen by the consistent results in Table 2, such uncertainty is only expected to be marginally larger.
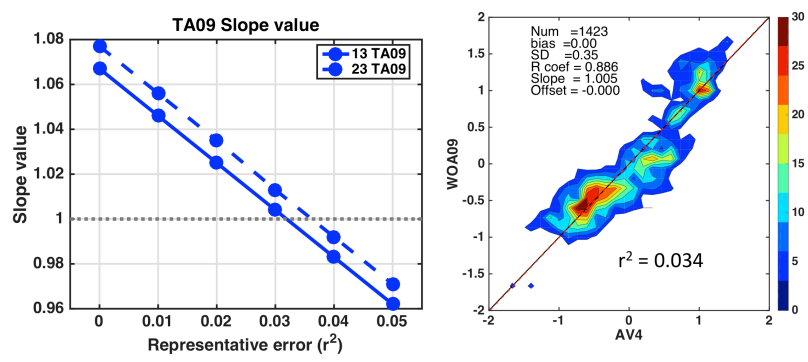

Fig. 4. The left plot represents the slope values as a function of representativeness error $\left(\mathrm{r}^{2}\right)$ for the triplet TAO-AV4-WOA09 (TA09). The blue solid (dashed) line corresponds to the slope values of the scatterplot TAO/WOA09 (AV4/WOA09). The right plot shows the scatterplot of AV4 versus WOA09 after TC, using a representativeness error, $r^{2}$, of 0.034. Same legend as that of Fig. 2.

\section{B. Error Assessment}

The estimated $r^{2}$ values in Table 2 are used in this section to estimate the random errors of each individual SSS data product at the satellite resolved scales. As explained in section III, using eqs. (3)-(5) (the latter with $K=0$ ), TC provides error values at the scale resolved by system 3 . To obtain the error variances on the scale of system $2, r^{2}$ has to be subtracted from both the system 1 and the system 2 error variances, and added to that of system 3 . For example, with the $\delta_{i}^{2}$ derived from the triplet TA09, the SD errors for TAO, AV4, and WOA09 at AV4 scale can be estimated as: $\delta_{T A O}=\sqrt{\delta_{1}^{2}-0.034}$; $\delta_{A V 4}=\sqrt{\delta_{2}^{2}-0.034}$; and $\delta_{\text {WOA09 }}=\sqrt{\delta_{3}^{2}+0.034}$.

Also, as discussed in section IV.A, AV4 resolves smaller scales than SOA. Therefore, the error assessment is carried out separately at both resolutions.

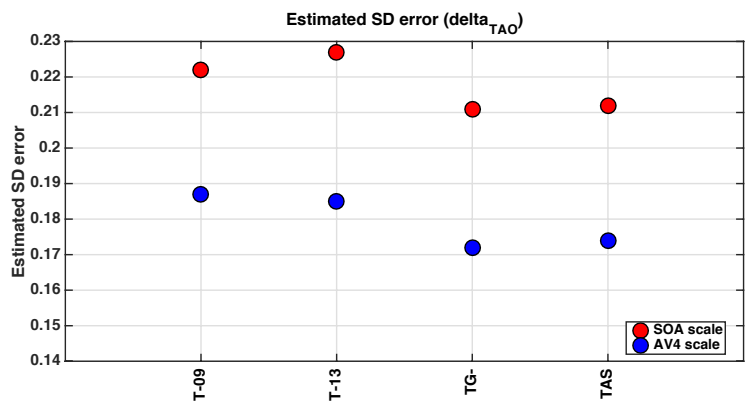

Fig. 5. Estimated TAO SD error $\left(\delta_{T A O}\right)$ estimated by the TC algorithm at both the SOA scale (red points) and the AV4 scale (blue points) for the following triplets: TA09 and TS09 (denoted as T-09), TA13 and TS13 (as T13), TGA and TGS (as TG-), and TAS.

Figure 5 shows the TAO random errors as estimated by the seven different triplets, both at AV4 (blue circles) and SOA (red circles) scales. The TAO errors, when evaluated at the same resolution (either AV4 or SOA) should always be the same. As such, the TAO error variation seen in Figure 5 gives an indication of the uncertainty of the proposed methodology (i.e., about 0.01). Also, note that since AV4 resolves smaller scales than SOA, the TAO errors at AV4 scales should be smaller than those at SOA scales (i.e., the TAO small-scale SSS variability should be more compatible with AV4 scales than with SOA scales). This is true for all the triplet combinations (see Figure 5), which indicates once again that the TC results are indeed consistent.

\begin{tabular}{c|c|c|c|c|c|c}
\hline \multicolumn{7}{|c|}{ Errors at AV4 scale } \\
\hline Triplet & TAO & GLORYS2V3 & AV4 & SOA & WOA13 & WOA09 \\
\hline TA09 & 0.187 & & 0.158 & & & 0.314 \\
\hline TA13 & 0.185 & & 0.162 & & 0.290 & \\
\hline TAS & 0.174 & & 0.173 & 0.244 & & \\
\hline TGA & 0.172 & 0.175 & 0.175 & & & \\
\hline $\begin{array}{c}\text { Mean } \\
\text { error }\end{array}$ & 0.178 & 0.175 & 0.167 & 0.244 & 0.290 & 0.314 \\
\hline
\end{tabular}

Table 3: Estimated SD errors of the different salinity measurements at AV4 scale.

\begin{tabular}{c|c|c|c|c|c|c}
\hline \multicolumn{7}{|c|}{ Errors at SOA scale } \\
\hline Triplet & TAO & GLORYS2V3 & AV4 & SOA & WOA13 & WOA09 \\
\hline TS09 & 0.222 & & & 0.201 & & 0.293 \\
\hline TS13 & 0.227 & & & 0.193 & 0.257 & \\
\hline $\boldsymbol{T A S}$ & 0.212 & & 0.211 & 0.212 & & \\
\hline TGS & 0.211 & 0.208 & & 0.210 & & \\
\hline $\begin{array}{c}\text { Mean } \\
\text { error }\end{array}$ & 0.218 & 0.208 & 0.211 & 0.204 & 0.257 & 0.293 \\
& & & & &
\end{tabular}

Table 4: Estimated SD errors of the different salinity measurements at SOA scale.

The estimated random error for each individual source and triplet combination at the AV4 and SOA resolved scales are shown in Tables 3 and 4, respectively. In these tables, the last row shows the resulting error average (note that for some products, only one value is actually estimated). As already mentioned, triplets including climatology provide somewhat less reliable error estimates. As such, one may also exclude those triplets from the analysis and focus on the more reliable ones, i.e., TGA, TGS, and TAS. By doing so though, the error estimates for TAO, GLORYS2V3, AV4, and SOA do not significantly vary.

It can be concluded that, at AV4 scales, the AV4 product shows the smallest random errors (0.17), although similar to those of GLORYS2V3 and TAO (0.18), while the SOA product shows relatively larger error $(0.24)$. However, at SOA scales, the SOA product shows the smallest errors $(0.20)$, although similar to those of GLORYS2V3 (0.21), AV4 (0.21), and TAO (0.22). The climatology, as expected, shows the largest random errors at both the AV4 and the SOA scales, although the uncertainty in the error estimates is somewhat larger due to the already discussed need for a higher order calibration. The estimated $r^{2}$ values represent an addition or subtraction of about $15-50 \%$ of the final error values, thus 
confirming the need to account for them in the triple collocation analysis.

\section{CONCLUSIONS}

The representativeness error has been accounted for during the triple collocation validation of six different SSS products along the tropical band. The validation has been carried out at the scales resolved by the two satellite (Aquarius and SMOS) products. It has been found that the representativeness error represents between $15 \%$ and $50 \%$ of the error estimates of the different products.

The six salinity products assessed in this study are: i) the in situ data from the Global Tropical Moored Buoy Array (TAO), ii) the GLORYS2V3 ocean reanalysis output provided by Copernicus; iii) the high-level salinity maps derived from the Aquarius (AV4) and SMOS (SOA) missions, and iv) the monthly climatologies provided by the World Ocean Atlas (WOA09 and WOA13). The analysis is performed in the tropics over the year 2013.

The Triple Collocation (TC) technique, as first introduced by [16], consists of simultaneously intercalibrating three independent, collocated data sources (triplets) and providing an estimate of their individual random errors. Note that, in this study, TAO data are used as calibration reference. TC accounts for products with different spatio-temporal characteristics and provides the output (random errors) at the scales resolved by the lowest resolution system, i.e., system 3 . The representativeness error, i.e., the true variance resolved by the relatively high resolution systems but not by the relatively low resolution system, therefore needs to be estimated, which in turn, allows providing the error estimates at different resolutions. In this study, all error estimates are provided at the spatio-temporal scales resolved by the satellite products, i.e., AV4 and SOA.

A method based on the intercalibration capabilities of TC has been used to estimate the representativeness error for each triplet. The representativeness error value that leads to the most optimal intercalibration of a particular triplet is then used to estimate the random errors for each individual data product for that triplet. Two relevant aspects of the TC methodology are used here to consolidate the validation results.

On the one hand, the sorting of the triplets is such that the lowest resolution system has to be system 3 . When system 3 is not the system with the lowest resolution, the computation of $r^{2}$ fails (i.e., it leads to negative random error values). As such, the products can be sorted in terms of their respective resolving spatio-temporal scales. Besides the in situ TAO, which has the highest spatio-temporal resolution, from finer to coarser, the order is GLORYS2V3, satellite (AV4 and SOA) and climatology (WOA13 and WOA09).

On the other hand, provided that the error estimates are given at a fixed spatio-temporal resolution (i.e., the satellite scales), the error estimate of a particular data product (e.g., GLORYS2v3) should always be the same regardless of the triplet combination used in TC. As such, several triplet combinations of the six different salinity products are used to check the consistency of the TC error output and provide an indication of the uncertainty in the error estimation for each individual product.

The TC analysis shows that the resolved scales of AV4 are significantly shorter than those of SOA. Although the Aquarius footprint is smaller than that of SMOS, the Objective Analysis (OA) applied to SMOS, with large correlation radii (see section II.A) to mitigate its poor radiometric accuracy, leads to a relatively coarse spatio-temporal resolution product. As such, the error assessment is carried out separately at both AV4 and SOA resolved scales. At AV4 scales, it is found that AV4 has an error of 0.17 , significantly smaller than that of SOA, i.e., about 0.24. In fact, AV4 outperforms all other products, although the ocean reanalysis (GLORYS2V3) and TAO show similar scores (i.e., 0.18). However, at SMOS scales, the SOA product $(0.20)$ outperforms all other products, although its error is similar to that of GLORYS2V3, AV4, and TAO. This opens the door for the verification of higher resolution SMOS-derived salinity products, such as the global and regional debiased non-Bayesian, available at http://bec.icm.csic.es [35] The WOA products show the highest errors at satellite scales. However, these error estimates have slightly larger uncertainty due to the fact that the WOA products cannot be linearly calibrated against the other salinity sources. Higher order calibration may lead to (slightly) more accurate assessment of the quality of the climatology maps.

Finally, the error of TAO at the AV4 and SOA spatiotemporal scales is 0.18 and 0.22 , respectively. Since the "truth" is defined as the ocean salinity at $1 \mathrm{~cm}$ depth and at the satellite-resolved spatio-temporal scales, these error values should include the contribution of the scale difference between the pointwise observation and the 0.25-1 degree cells of the satellite products, the vertical mismatch between the TAO measurement at 1-1.5 meters depth and the satellite retrievals at $1 \mathrm{~cm}$ (see stratification effects in, e.g., [36]), and the different temporal resolution of TAO (1 day) and the satellite products (7-9 days). The partition of these error contributions (notably the horizontal and vertical resolution mismatch) remains a research topic in oceanography.

Potential application of the TC developed here can help to understand the different processes (diffusivity, etc.) that take place in different ocean regions. Furthermore, this technique can be applied to longer time series to assess the quality of the salinity products in terms of interannual variability.

\section{ACKNOWLEDGMENT}

This study was founded by the Spanish $\mathrm{R}+\mathrm{D}$ plan under projects PROMISES (ESP2015-67549-C3-2) and L-BAND (ESP2017-89463-C3-1-R). The authors would like to thanks the SMOS BEC team for their inputs and help with the data processing. The Aquarius and SMOS products used here in this study can be found at http://podaac-opendap.jpl.nasa.gov and http://bec.icm.csic.es. GLORYS2V3 reanalysis have been downloaded from the Copernicus Marine Environment 
Monitoring Service (CMEMS, http://marine.copernicus.eu). We acknowledge the TAO project office for delivering the data of the Global Tropical Moored Buoy Array. We acknowledge the Ocean Climate Laboratory of NOAA for the World Ocean Atlas data. The authors would also like to thank the editor and the two anonymous reviewers for their help to improve the quality of this manuscript.

\section{REFERENCES}

[1] A.H. Taylor, and J.A. Stephens, "Seasonal and year to year variations in surface salinity at nine North Atlantic Ocean weather stations", Ocean. Acta, vol. 3(4), pp. 421-430, 1980.

[2] T. Delcroix, M. J. McPhaden, A. Dessier, and Y. Gouriou, "Time and space scales for sea surface salinity in the tropical oceans", Deep-Sea Res. I, vol. 52, pp. 787-813, 2005.

[3] G. Reverdin, E. Kestenare, C. Frankignoul, and T. Delcroix, "Surface salinity in the Atlantic Ocean (30S-50N)", Prog. Oceanogr., vol. 73, pp. 311-340, 2007.

[4] J. Mignot, and C. Frankignoul, "On the interannual variability of surface salinity in the Atlantic", Clim. Dyn., vol. 20, pp. 555-565, 2003.

[5] V. González-Gambau, A. Turiel, E. Olmedo, J. Martínez, I. Corbella, and A. Camps, "Nodal sampling, a new image reconstruction algorithm for SMOS", IEEE T. Geosci. Remote, vol. 54(4), pp. 23142328, 2016a. DOI: 10.1109/TGRS.2015.2499324.

[6] V. González-Gambau, E. Olmedo, A. Turiel, J. Martínez, J. Ballabrera-Poy, M. Portabella, and M. Piles, "Enhancing SMOS brightness temperature over the ocean using the nodal sampling image reconstruction technique", Rem. Sens. Environ., vol. 180, pp. 205-220, 2016b. DOI: 10.1016/j.rse.2015.12.032

[7] Umbert, M., Guimbard, S., Lagerloef, G., Thompson, L., BallabreraPoy, J., Portabella, M., and Turiel, A., "Detecting the surface salinity signature of Gulf Stream cold-core rings in Aquarius synergistic products," J. Geophys. Res. Oceans, vol. 20 (2), pp. 859-874, 2015 https://doi.org/10.1002/2014JC010466.

[8] Boutin, J., N. Martin, N. Kolodziejczyk, and G. Reverdin, "Interannual anomalies of SMOS sea surface salinity", Rem. Sens. Environ., vol. 180, pp. 128-263, 2016. doi:http://dx.doi.org/10.1016/j.rse.2016.02.053.

[9] E. Olmedo, J. Martínez, M. Umbert, N. Hoareau, M. Portabella, J. Ballabrera-Poy, and A. Turiel, "Improving time and space resolution of SMOS salinity maps using multifractal fusion", Rem. Sens. Environ., vol. 180, pp. 246-263, 2016.

[10] N. Hoareau, A. Turiel, M. Portabella, J. Ballabrera-Poy, and J. Vogelzang, "Singularity power spectra: a new method to improve the characterization of the geophysical consistency of gridded products. Application to sea surface salinity remote sensing maps", IEEE Trans. Geosci. Rem. Sens., under review, 2017.

[11] J. Boutin, N. Martin, X. Yin, J. Font, N. Reul and P. Spurgeon, "First Assessment of SMOS Data Over Open Ocean: Part II: Sea Surface Salinity", IEEE T. Geosci. Remote, vol. 50(5), pp. 1662-1675, 2012. DOI: $10.1109 /$ tgrs.2012.2184546.

[12] N. Reul, J. Terenelli, J. Boutin, B. Chapron, F. Paul, E. Brion, et al., "Overview of the First SMOS sea surface salinity products. Part I: Quality assessment for the second half of 2010", IEEE T. Geosci. Remote, vol. 50(5), pp. 1636-1647, 2012.

[13] O. Hernandez, J. Boutin, N. Kolodziejcyk, G. Reverdin, N. Martin, F. Gaillard, N. Reul, and J.L. Vergely, "SMOS salinity in the subtropical North Atlantic salinity maximum: 1. Comparison with Aquarius and in situ salinity", J. Geophys. Res., vol. 119, pp. 8878-8896, 2014. DOI: 10.1002/2013JC009610.

[14] N. Kolodziejczyk, O. Hernandez, J. Boutin, and G. Reverdin, “ SMOS salinity in the subtropical North Atlantic salinity maximum: 2.
Two-dimensional horizontal thermohaline variability", J. Geophys. Res., vol. 120, pp. 972-987, 2015. DOI: 10.1002/2014JC010103.

[15] W. Tang, A. Fore, S. Yueh, T. Lee, A. Hayashi, A. Sanchez-Franks, J. Martinez, B. King, and D. Baranowski, "Validating SMAP SSS with in situ measurements", Rem. Sens. Environ.,vol. 200, pp. 326340, 2017. DOI: $10.1016 /$ j.rse.2017.08.021.

[16] A. Stoffelen, "Error modeling and calibration: towards the true surface wind speed", J. Geophys. Res., vol. 103, pp. 7755-7766, 1998. DOI: $10.1175 / 2010 J C L I 3294.1$.

[17] M. Portabella and A. Stoffelen, "On scatterometer Ocean Stress", J. Atm. Ocean. Tech., vol. 26(2), pp. 368-382, 2009. DOI: 10.1175/2008JTECHO578.1

[18] J. Vogelzang, A. Stoffelen, A. Verhoef, and J. Figa-Saldaña, "On the quality of high-resolution scatterometer winds", J. Geophys. Res., vol. 116, C10033, 2011. DOI: 10.1029/2010JC006640.

[19] W. Lin, M. Portabella, A. Stoffelen, J. Vogelzang, and A. Verhoef, "On mesoscale analysis and ASCAT ambiguity removal", Q. J.R. Meteor. Soc., vol. 142, pp. 1745-1756, 2016.

[20] W.B. Anderson, B.F. Zaitchik, C.R. Hain, M.C. Anderson, M.T. Yilmaz, G. Mecikalski, L. Schultz, " Towards an integrated soil moisture drought monitor for East Africa" Hydrol. Earth Syst. Sci., vol. 16, pp. 2893-2913, 2012.

[21] C. Draper, R. Reichle, R. de Jeu, V. Naeimi, R. Parinussa, and W. Wagner, "Estimating root mean square errors in remotely sensed soil moisture over continental scale domains", Rem. Sens. Environ., vol. 137, pp. 288-298, 2013.

[22] R.A. Roebeling, E.L. Wolters, J.F. Meirink, and H. Leijnse, "Triple collocation of summer precipitation retrievals from SEVIRI over Europe with gridded rain gauge and weather radar data" $J$. Hydrometeorol., vol. 13(5), pp. 1552-1566, 2012. DOI: 10.1175/JHM-D-11-089.1.

[23] S. Ratheesh, B. Mankad, S. Basu, R. Kumar, and R. Sharma, "Assessment of satellite-Derived Sea Surface Salinity in the Indian Ocean", IEEE Geos. and Rem. Sens. Letters, vol. 10, pp. 428-431, 2013.

[24] W. Lin, M. Portabella, A. Stoffelen, J. Vogelzang, and A. Verhoef, "ASCAT wind quality under high subcell wind variability conditions", J. Geophys. Res. Oceans, vol. 120(8), pp. 5804-5819, 2015.

[25] UNESCO "The Practical Salinity Scale 1978 and the International Equation of State of Seawater 1980", United Nations Educational, Scientific and Cultural Organization, Paris, France, UNESCO Technical Papers in Marine Science Number 36, 10th report of the Joint Panel on Oceanographic Tables and Standards, 1981.

[26] S. Mecklenburg, M. Drush, Y.H. Kerr, J. Font, M. Martin-Neira, S. Delwart, G. Buenadicha, N. Reul, E. Daganzo-Eusebio, R. Oliva, and R. Crapolicchio, "ESA's Soil Moisture and Ocean Salinity Mission: Mission performance and Operations", IEEE T. Geosci. Remote, vol. 50, pp. 1354-1366, 2012. DOI: 10.1109/TGRS.2012.2187666.

[27] BEC, "SMOS-BEC Ocean and Land Products Description BECSMOS-0008-QR", BEC. version 1.0, 2016. Available: http://bec.icm.csic.es/doc/BEC-SMOS-0008-QR.pdf.

[28] M.M. Zweng, J.R. Reagan, J.I. Antonov, R.A. Locarnini, A.V. Mishonov, T.P. Boyer, H.E. Garcia, O.K. Baranova, D.R. Johnson, D. Seidov, and M.M. Biddle, "World Ocean Atlas 2013, Volume 2: Salinity", S. Levitus, Ed., A. Mishonov Technical Ed.; NOAA Atlas NESDIS 74, 2013.

[29] M. Madec and NEMO team, "NEMO ocean engine, version 3.4", Note du Pôle de modélisation de l'Institut Pierre-Simon Laplace, No 27, ISSN, pp. 1288-1619, 2012.

[30] L. Parent, N. Ferry, B. Barnier, G. Garric, C. Bricaud, C.E. Testut, O. Le Galloudec, J. M. Lellouche, E. Greiner, M. Drevillon, E. Rémy, J. M. Molines, S. Guinehut, and C. Cabanes, "GLOBAL eddypermitting ocean reanalysis and simulations of the period 1992 to present", Venice, Italy: Proceeding of the "20 years of progress in radar altimetry”, Sept., 24-29, 2012.

[31] J. I. Antonov, D. Seidov, T.P. Boyer, R.A. Locarnini, A.V. Mishonov, H.E. Garcia, O.K. Baranova, M.M. Zweng, and D.R. Johnson, "World Ocean Atlas 2009, Volume 2: Salinity", S. Levitus, Ed. NOAA 
Atlas NESDIS 69, U.S. Government Printing Office, Washington, D.C: S. Levitus, Ed. NOAA Atlas NESDIS 69, U.S. Government Printing Office, 2010.

[32] W. Lin, M. Portabella, A. Stoffelen, A. Verhoef, and A. Turiel, "ASCAT wind quality control near rain," IEEE Trans. Geosci. Rem. Sens., vol. 53(8), pp. 4165-4177, 2015. DOI: 10.1109/TGRS.2015.2392372.

[33] Vogelzang, G. P. King, and A. Stoffelen, "Spatial variances of wind fields and their relation to second-order structure functions and spectra", J. Geophys. Res. Oceans, vol. 120, pp. 1048-1064, 2015. DOI: $10.1002 / 2014 J C 010239$.

[34] W. Lin, and M. Portabella, "Toward an improved wind quality control for RapidScat", IEEE T. Geosci. Remote Sens., vol. 55(7), pp. 3922-3930, 2017. DOI: 10.1109/TGRS.2017.2683720.

[35] Olmedo, E., Martínez, J., Turiel, A., Ballabrera-Poy, J., and Portabella, M., "Debiased Non-Bayesian retrieval: a novel approach to SMOS Sea Surface Salinity," Remote Sensing of Environment, 193 pp. 103-126, https://doi.org/10.1016/j.rse.2017.02.023, 2017.

[36] Boutin, J., Y. Chao, W.E. Asher, T. Delcroix, R. Drucker, K. Drushka, N. Kolodziejczyk, T. Lee, N. Reul, G. Reverdin, J. Schanze, A. Soloviev, L. Yu, J. Anderson, L. Brucker, E. Dinnat, A.S. Garcia, W.L. Jones, C. Maes, T. Meissner, W. Tang, N. Vinogradova, B. Ward, "Satellite and In Situ Salinity: Understanding Near-surface Stratification and Sub-footprint Variability," Bull. Amer. Meteor. Soc. DOI: 10.1175/BAMS-D-15-00032.1, 2015. 


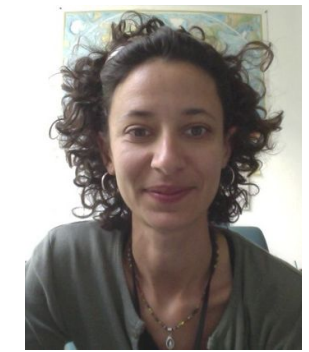

Nina Hoareau was born on August 13 of 1983 in France. She received a B.Sc degree in Physical Oceanography in 2007 from University of Marseille; and a M.Sc. degree in Climatology in 2009 from the University of Paul Sabatier (Toulouse III). She was at the Institut de Ciències del Mar (ICM-CSIC), Barcelona, into the BEC team and working on the validation of the SMOS satellite.

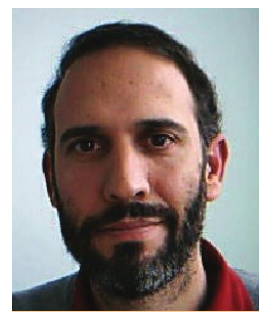

Marcos Portabella was born on October 14, 1970 in Spain. He received the B.Sc. degree in Physics in1994 from the University of Barcelona, Barcelona, Spain; the M.Sc. in Remote Sensing in 1995 from the Institute of Space Studies of Catalonia, Barcelona,Spain; and the Ph.D. degree in Physics in 2002, fromthe University of Barcelona, Spain. He is currently with the Institut de Ciències del Mar (ICM-CSIC), Barcelona, working on satelliteremote sensing. In particular, he is involved in scatterometry and Lband radiometry.

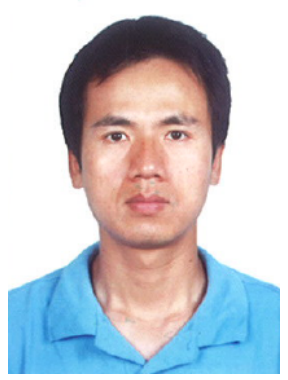

Wenming Lin was born on April 22, 1984, in China. He received the B.Sc. degree in engineering from Wuhan University, Wuhan, China, in 2006 and the Ph.D. degree in engineering from the Center for Space Science and Applied Research, Chinese Academy of Sciences, Beijing, China, in 2011. He was a Postdoctoral Researcher with the Institut de Ciències del Mar (ICM-CSIC), Barcelona, Spain, working on the scatterometer wind quality control from 2011-2017. $\mathrm{He}$ is currently working with University of Information Science and Technology (NUIST), Nanjing, China.

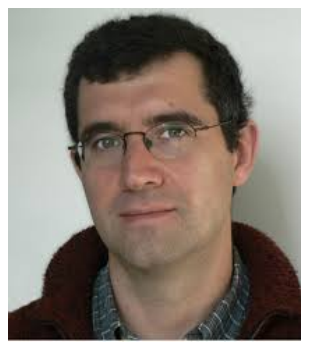

Joaquim Ballabrera-Poy was born on March 30, 1966, in Barcelona, Spain. He received the B.Sc. degree from the University of Barcelona,
Barcelona, in 1991 and the Dyplôme d'Études Avancées (DEA) and the Ph.D. degree in geophysics from Joseph Fourier University, Grenoble, France. He is currently with the Institut de Ciències del Mar (ICM-CSIC), Barcelona, working on numerical modeling, data assimilation, and remote sensing.

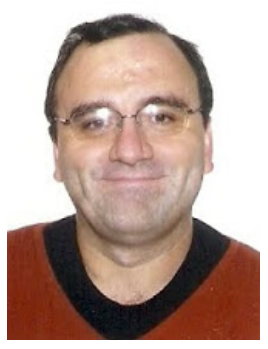

Antonio Turiel was born on May 1, 1970, in Spain. He received the B.Sc. degree in physics, the B.Sc. degree in mathematics, and the Ph.D. degree in theoretical physics from the Autonomous University of Madrid, Madrid, Spain, in 1993, 1994, and 1998, respectively. He is currently with the Department of Physical Oceanography, Institut de Ciències del Mar (ICM-CSIC), Barcelona. His research topics include signal and image processing applied to remote sensing of the oceans, marine turbulence at mesoscale, and ocean circulation at different scales. 